\title{
Fitness evaluation of two Brazilian Aedes aegypti field populations with distinct levels of resistance to the organophosphate temephos
}

\author{
Thiago Affonso Belinato ${ }^{1,2}$, Ademir Jesus Martins ${ }^{1,2}$, Denise Valle $e^{1,2,3 /+}$ \\ ${ }^{1}$ Laboratório de Fisiologia e Controle de Artrópodes Vetores, Instituto Oswaldo Cruz-Fiocruz, Rio de Janeiro, RJ, Brasil \\ ${ }^{2}$ Laboratório de Entomologia, Instituto de Biologia do Exército, Rio de Janeiro, RJ, Brasil ${ }^{3}$ Instituto Nacional de Ciência e Tecnologia em \\ Entomologia Molecular, Instituto de Bioquímica Médica, Universidade Federal do Rio de Janeiro, Rio de Janeiro, RJ, Brasil
}

In Brazil, decades of dengue vector control using organophosphates and pyrethroids have led to dissemination of resistance. Although these insecticides have been employed for decades against Aedes aegypti in the country, knowledge of the impact of temephos resistance on vector viability is limited. We evaluated several fitness parameters in two Brazilian Ae. aegypti populations, both classified as deltamethrin resistant but with distinct resistant ratios (RR) for temephos. The insecticide-susceptible Rockefeller strain was used as an experimental control. The population presenting the higher temephos resistance level, Aparecida de Goiânia, state of Goiás (RR ${ }_{95}$ of 19.2), exhibited deficiency in the following four parameters: blood meal acceptance, amount of ingested blood, number of eggs and frequency of inseminated females. Mosquitoes from Boa Vista, state of Roraima, the population with lower temephos resistance level ( $R R_{05}$ of 7.4), presented impairment in only two parameters, blood meal acceptance and frequency of inseminated females. These results indicate that the overall fitness handicap was proportional to temephos resistance levels. However, it is unlikely that these disabilities can be attributed solely to temephos resistance, since both populations are also resistant to deltamethrin and harbour the $\mathrm{kdr}$ allele, which indicates resistance to pyrethroids. The effects of reduced fitness in resistant populations are discussed.

Key words: Aedes aegypti - insecticide resistance - temephos - fitness

Aedes aegypti (L., 1762) is a mosquito with wide geographic distribution, predominantly in tropical and subtropical regions (Jansen \& Beebe 2010). The females feed preferentially on humans and are strongly associated with the urban environment (Gubler 2002, Ponlawat \& Harrington 2005, Siriyasatien 2010). This species is considered the main dengue vector, both its global distribution and the number of dengue cases having expanded since the 1950s (Mackenzie et al. 2004).

Insecticides still play a major role in the control of this mosquito, especially the organophosphate (OP) temephos which was the sole larvicide recommended for use in drinking water for a long period (Chavasse \& Yap 1997). In Brazil, this pesticide has been employed since 1967 against Ae. aegypti larvae and its use was intensified after the 1986 dengue outbreak (Braga \& Valle 2007a). Control of Ae. aegypti in the country was accomplished with OPs until 2000-2001, when pyrethroids were introduced (da-Cunha et al. 2005, Montella et al. 2007).

Vector control strategies based on intense and frequent insecticide applications result in increased frequency of resistance in a given population, a situation that culminates with the impairment or even complete failure of vector control. Resistance to temephos, as well as to other

Financial support: IOC-FIOCRUZ, MS/SVS, PRONEX-Rede Dengue/MCT, CNPq, DECIT/SCTIE/MS, FAPERJ

+ Corresponding author: dvalle@ioc.fiocruz.br

Received 14 November 2011

Accepted 12 April 2012 insecticide classes, such as pyrethroids, has already been detected in several Brazilian Ae. aegypti field populations (Macoris et al. 1999, Lima et al. 2003, Braga et al. 2004, da-Cunha et al. 2005, Montella et al. 2007). Both OP and pyrethroid insecticides act on the insect central nervous system. OPs bind to the enzyme acetylcholinesterase, while pyrethroids act on voltage-gated sodium channels.

Main resistance mechanisms include modifications in the insecticide target site, which can hamper or prevent insecticide binding. Pyrethroids, for example, keep the sodium channels across axons in the open conformation, resulting in repetitive nerve impulses leading to paralysis, an effect known as "knockdown". Pyrethroidresistant insects exhibit modified sodium channels and are referred to as "knockdown resistant", or $k d r$ (Martins \& Valle 2012). Acetylcholinesterase hydrolyses the neurotransmitter acetylcholine, removing it from the synapse and interrupting impulse propagation. In susceptible insects, OP binding to this enzyme impedes its function, resulting in continuous transmission of nerve impulses (Hemingway \& Ranson 2000, Ranson et al. 2004).

Another major resistance mechanism is activation of insect xenobiotic detoxification pathways, also known as metabolic resistance. Three groups of enzymes are involved in this process: esterases, glutathion-s-transferases and mixed function oxidases (Hemingway \& Ranson 2000).

Resistant insects are supposed to exhibit a great adaptive advantage in an environment exposed to frequent or continuous insecticide pressure. Nevertheless, insecticide resistance is related to an energetic cost that can influence mosquito biology in the field. According to Roush and McKenzie (1987), fitness costs can be considered a 
consequence of trade-offs between the allocation of energy underlying insecticide resistance mechanisms and insect fitness. As a result, when insecticide use is interrupted, resistant individuals tend to be less competitive compared to susceptible individuals, leading to decreasing frequencies of resistant insects over time. However, in certain situations, resistance can be unrelated to development or reproduction. Moreover, resistance can even display a positive effect, resulting in an adaptive advantage under field conditions (Rivero et al. 2010).

Several parameters of insect biology can be affected by pesticide resistance, such as development time, adult longevity, behaviour, reproduction and the immune system (Berticat et al. 2002, 2004, Rivero et al. 2010). Once altered, these aspects have the potential to influence both the dynamics of insecticide resistance dissemination and the relationship among vectors and the parasites they transmit. To address this correlation, the present study evaluated a series of life table parameters in two natural Ae. aegypti populations showing distinct levels of resistance to the OP temephos.

\section{MATERIALS AND METHODS}

Mosquitoes - Two populations of Brazilian Ae. aegypti (Fig. 1), Boa Vista, state of Roraima and Aparecida de Goiânia, state of Goiás, were chosen according to their levels of resistance to OP temephos, a major larvicide adopted for control of this mosquito. The Boa Vista (BVT) F2 and Aparecida de Goiânia (APG) F1 mosquitoes used in the assays were derived from eggs collected in 2007 and 2008, respectively. Mosquitoes from the Rockefeller (Rock) strain, which served as a reference lineage for both susceptibility and vigour, were also analysed as the control group (Kuno 2010).

Mosquito rearing - To synchronise development, eggs were allowed to hatch during $1 \mathrm{~h}$. Groups of 1,000 first instar larvae were then transferred to plastic basins (33 $\times 24 \times 8 \mathrm{~cm})$ containing $1 \mathrm{~L}$ dechlorinated water and $1 \mathrm{~g}$ cat food (Friskies ${ }^{\circledR}$, Purina, São Paulo, SP) and were maintained in a biological oxygen demand (BOD) incubator at $26^{\circ} \mathrm{C}$. When rearing proceeded until the adult stage, food was replaced every three days. Adult mosquitoes were maintained in an insectary at $26 \pm 1^{\circ} \mathrm{C}$ under $80 \pm 10 \%$ relative humidity (rh).

Bioassays - Temephos resistance levels were evaluated in larvae from both populations through dose response bioassays (WHO 1981). In each assay 10 insecticide concentrations prepared with Temephos PESTANAL ${ }^{\circledR}$ (Sigma-Aldrich Brazil, São Paulo, SP) were tested. For each concentration, there were four replicates, each with 20 third instar larvae in $100 \mathrm{~mL}$ solution. Lethal concentrations (LCs) were calculated via probit analysis (software Polo-PC, LeOra Software, Berkeley, CA) (Raymond 1985). Resistance ratios $\left(R_{50}\right.$ and $\left.R R_{95}\right)$ were obtained by dividing the LC of the field population (BVT or APG) by the equivalent $\mathrm{LC}$ from the Rock strain.

Deltamethrin resistance was also evaluated via the WHO (1998) protocol with slight modifications using impregnated papers. Adult females, one to three days old, non blood fed, were exposed to a deltamethrin diagnostic dose $\left(3.65 \mathrm{mg} / \mathrm{m}^{2}\right)$, previously calibrated with Rock mosquitoes. It corresponded to twice the minimal dose resulting in total mortality of Rock strain after a $1 \mathrm{~h}$ exposure and $24 \mathrm{~h}$ recovery, as recommended for this assay. Each test consisted of three replicates using deltamethrin-impregnated paper plus one control, without the insecticide. For each replicate, 15-20 females were used.

Molecular assays - The allele-specific polymerase chain reaction (PCR)-based genotyping strategy was applied to investigate the frequency of the Val1016Ile $k d r$ substitution in the Ae. aegypti voltage-gated sodium channel gene $\left(A a N a_{V}\right)$ in BVT and APG populations. The protocol for genomic DNA extraction as well as the PCR conditions and subsequent analysis were according to Martins et al. (2009).

Adult longevity - Groups of 15 males and 15 females from each field population and the Rock strain were transferred to small carton cages $(8.5 \mathrm{~cm}$ diameter and $8.5 \mathrm{~cm}$ high) and fed ad libitum with a $10 \%$ sugar solution. Mortality of each gender was recorded daily.

Blood meal acceptance and amount of ingested blood - Adult females three to five days old, collected in a cage containing males, were put in contact with a ketamineanaesthetised guinea pig (Hawk \& Leary 1995) for 30 min and the number of females that successfully fed on blood was recorded. To determine the amount of blood ingested, we calculated the difference between the average weights of three groups each of 10 non-fed and

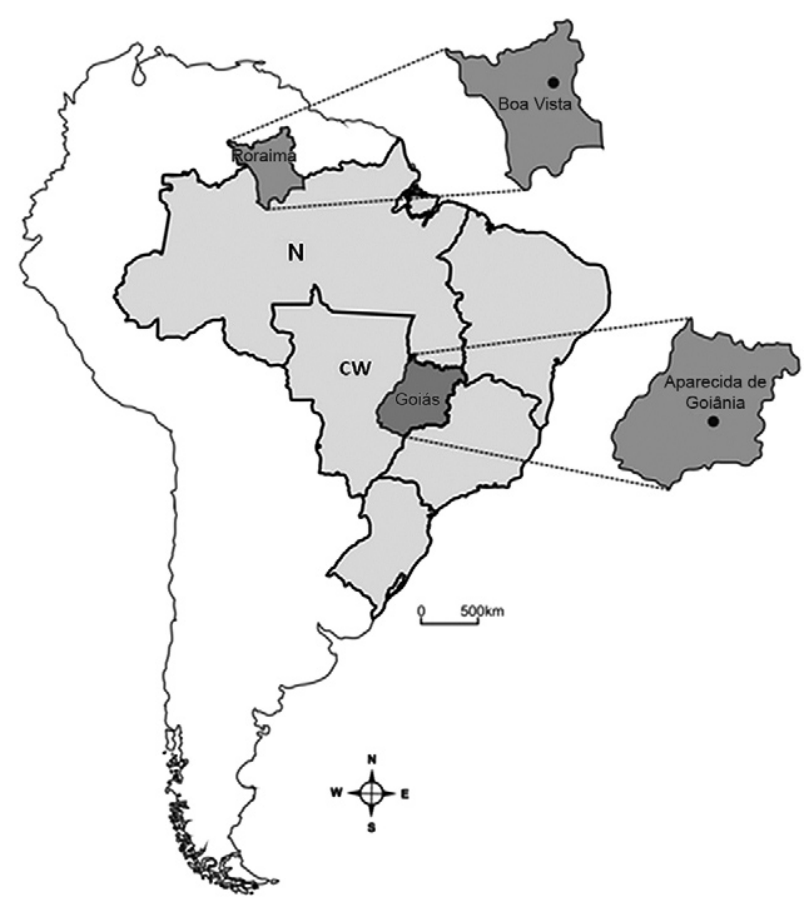

Fig. 1: Brazilian map indicating regions, states and the geographical location of Aedes aegypti populations under evaluation. The municipality of Boa Vista is located in the state of Roraima, in the North $(\mathrm{N})$ Region, and Aparecida de Goiânia in the state of Goiás, in the Central West (CW) Region of Brazil. 
10 blood-fed females, weighed on an analytical balance (APX-200, Denver instrument), as performed elsewhere (Belinato et al. 2009).

Egg laying - Three days after the blood meal, individual females were placed in inverted Petri dishes with the lid internally lined with a filter paper soaked with dechlorinated water (Valencia et al. 1996) and kept at $26^{\circ} \mathrm{C}$ in a BOD incubator. After $24 \mathrm{~h}$, females were removed and three parameters were recorded: the number of ovipositing females, the number and viability of eggs.

Frequency of inseminated females - For each population, 15 groups consisting of three females and one male each, all of which were two to three days old, were maintained together in transparent $50 \mathrm{~mL}$ Falcon tubes. All the specimens employed in this assay were reared individually since the pupa stage, in order to ensure the virginity of the adults. After three days, female spermathecae were dissected and the presence of spermatozoids was assessed with the aid of an optic microscope (Nikon Biophot, 200X).

Statistical analysis - All the experiments described herein were repeated at least three times. Data obtained for each parameter evaluated were compared using $t$ tests or $\chi^{2}$ analysis, as indicated in the results, except for longevity data that were subjected to Kruskal-Wallis followed by Dunn's Multiple Comparison Test. Graph-Pad Prism version 5.0 for Windows was adopted for all analyses (GraphPad Software, San Diego California USA) (graphpad.com).

\section{RESULTS}

Insecticide resistance status of field populations - Temephos produced a dose-response effect over the Rock strain and the two evaluated field populations. Table I shows LCs and resistance ratios obtained by the probit analysis. The APG population exhibited both higher temephos resistance ratio and greater heterogeneity. Nevertheless, both APG and BVT mosquitoes presented a high resistance status against this OP. In Brazil, Ae. aegypti populations with temephos $\mathrm{RR}_{95}$ above 3.0 are considered resistant and are subject to insecticide substitution (Ministry of Health, unpublished observations).

Resistance to the pyrethroid deltamethrin was also evaluated. Qualitative assays, performed with the deltamethrin diagnostic dose, detected $11.7 \%$ mortality in APG mosquitoes and $37.6 \%$ in BVT. According to WHO (Davidson \& Zahar 1973), mortality levels below $80 \%$ in these assays are indicative of resistance. Additionally, the $k d r$ mutation Val1016Ile was detected in both populations, as depicted in Table II.

Adult longevity - Statistical comparisons of female and male survivorship were performed arbitrarily on the 30th and 40th days following adult emergence. When each gender was compared, no differences in the longevity were noted among APG, BVT and the Rock strain on both days (Kruskal Wallis followed by Dunn's multiple comparison test, $p>0.05$ ). In all cases, including in the Rock strain, females survived slightly longer, male attaining total mortality when $4.5 \%$ of females were still alive (Fig. 2A, B).
Blood meal - Blood meal acceptance was significantly different between field populations and the Rock strain (Fig. 3A). In the latter, 96\% (86/90) of the females were able to feed. Significantly fewer insects from BVT $(73 / 90)\left(\chi_{0.05,1}^{2}=9.11 ; p=0.0013\right)$ and APG $(69 / 90)\left(\chi_{0.05,1}^{2}\right.$ $=13.42 ; \mathrm{p}=0.0001)$ accepted the blood meal. However, no differences between field populations were noted $\left(\chi_{0.05,1}^{2}=0,53 ; \mathrm{p}=0.2325\right)$.

When the amount of ingested blood was compared, that ingested by the APG population, which showed a higher level of temephos resistance, was significantly lower (by approximately 15\%) compared to either the Rock strain $\left(t_{0.05(1), 11} ; \mathrm{p}=0.008\right)$ or BVT mosquitoes $\left(t_{0.05(1), 16} ; \mathrm{p}=0.0087\right)$ (Fig. 3B). The amount of blood ingested by BVT females was $5 \%$ lower on average compared to Rock females, though this difference was not significant $\left(t_{0.05(1), 11}=1.253 ; \mathrm{p}=0.1181\right)$.

Number and viability of oviposited eggs - A relationship was observed between the volume of blood ingested by each population and the number of eggs. APG females laid significantly fewer eggs $(81 \pm 30)$ than Rock $\left(103 \pm 19, t_{0.05(1) 79}=4.096 ; \mathrm{p}<0.0001\right)$ and BVT females $\left(104 \pm 17, t_{0.05(1), 79}=4.393 ; \mathrm{p}<0.0001\right)$ (Fig. 4). The average number of eggs per APG female was $21 \%$ lower than for Rock. By contrast, no significant differences were found between the quantity of eggs laid by Rock and BVT females $\left(t_{0.05(1), 114}=0.2226 ; \mathrm{p}=0.4121\right)$. The

\section{TABLE I}

Susceptibility status of two Brazilian Aedes aegypti field populations, Boa Vista (BVT) and Aparecida de Goiânia (APG), to the organophosphate temephos, the sole larvicide employed in the country since 1967

\begin{tabular}{lccccc}
\hline Population & $\begin{array}{c}\mathrm{LC}_{50} \\
(\mathrm{ug} / \mathrm{L})\end{array}$ & $\mathrm{RR}_{50}$ & $\begin{array}{c}\mathrm{LC}_{95} \\
(\mathrm{ug} / \mathrm{L})\end{array}$ & $\mathrm{RR}_{95}$ & Slope \\
\hline Rock & 2.1 & - & 4.1 & - & 6.2 \\
BVT & 8.6 & 4.1 & 30.4 & 7.4 & 4.4 \\
APG & 32.9 & 15.7 & 78.7 & 19.2 & 3.1 \\
\hline
\end{tabular}

the reference strain Rockefeller (Rock) was used as the susceptibility control. LC: lethal concentration; RR: resistance ratio.

\section{TABLE II}

Frequency of $A a N a_{v}$ Val1016Ile $k d r$ mutation in two Brazilian Aedes aegypti populations

\begin{tabular}{lcc}
\hline Population & $\begin{array}{c}\text { APG } \\
\text { (F2) }\end{array}$ & $\begin{array}{c}\text { BVT } \\
\text { (F3) }\end{array}$ \\
\hline 1016Ile allelic frequency $^{a}$ & 0.293 & 0.067 \\
1016Ile/Ile genotypic frequency $^{a}$ & 0.103 & 0.033 \\
\hline
\end{tabular}

a: 29 and 30 individuals from respectively Aparecida de Goiânia (APG) and Boa Vista (BVT) were evaluated. 
viability of eggs was comparable for the three strains evaluated, all of which hatched at rates greater than $90 \%$ $\left(t_{0.05(1)} ; \mathrm{p}>0.05\right)$.

Frequency of inseminated females - At the end of the three-day mating period, $98 \%$ of the Rock males had inseminated their three available females (Fig. 5). The remaining $2 \%$ inseminated only two females. In contrast, there was a great reduction in the number of females inseminated by the field males. Only $54 \%$ of the BVT males successfully inseminated their three females, whereas $7 \%$ did not inseminate any female. The plight was even more pronounced for the APG males, among which only $7 \%$ successfully inseminated their three females and the majority $(68 \%)$ did not inseminate any female.

\section{DISCUSSION}

Presently the main Ae. aegypti control strategy relies upon chemical insecticides. In Brazil, OPs were widely adopted for the control of both larvae and adults of this mosquito, resulting in resistance dissemination throughout the country. In 2001, pyrethroids started to be employed against adults by the Brazilian dengue control program (Braga \& Valle 2007b). However, the dissemination of pyrethroid resistance was soon verified (da-Cunha et al. 2005), initially as a consequence of metabolic resistance (Montella et al. 2007) and later as a mutation in the insecticide target site (Martins et al. 2009). It is well known that in many cases one of the effects of insecticide resistance is the impairment of a series of mosquito fitness parameters. We investigated, in two field Ae. aegypti Brazilian populations, several viability parameters in order to evaluate the effect of resistance on vector fitness.

In this study, a slight difference in longevity was noted between males and females within each strain, but not between strains. There is great diversity among the results of previous reports assessing insecticide resistance and longevity, particularly with regard to the insecticide class or culicid species addressed. This may be explained in part by differences in methodology. Deltamethrin selection of Ae. aegypti or Culex pipiens pallens under laboratory conditions for nine (Martins et al. 2012) or 12 (Li et al. 2002) generations, respectively, was found to result in decreased longevity. In both cases, survival assays were performed with a sugar solution provided $a d$ libitum. In contrast, Hardstone et al. (2010) verified that Culex quinquefasciatus female adults resistant to the pyrethroid permethrin survived longer than susceptible ones when sustained with sugar. However, the authors did not find any differences in survival between males and females or between susceptible and resistant strains when adults were kept exclusively with water. In the latter case, the parameter evaluated was not longevity, but tolerance to starvation, with deprivation of energy resources derived from the immature stages. Agnew et al. (2004) reported greater vulnerability to starvation for three $C x$. quinquefasciatus strains homozygous for the ester $^{l}$, ester $^{4}$ or $A c e-1^{R}$ allele, all of which are related to
A

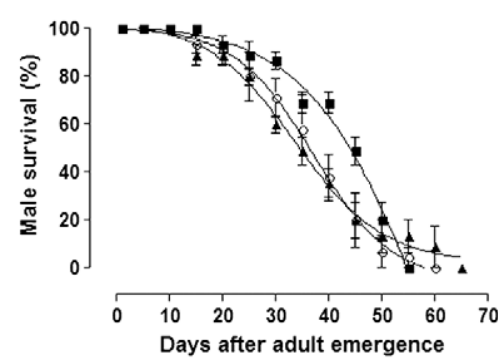

B

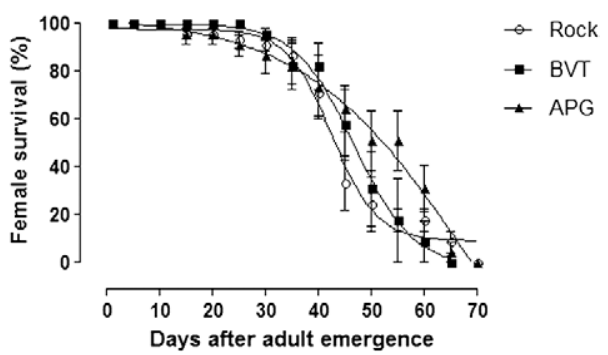

Fig. 2: non linear regression curves showing the longevity, registered every five days, of males (A) and females (B) from Rockefeller strain (Rock) and two field populations, Aparecida de Goiânia (APG) and Boa Vista (BVT).

A

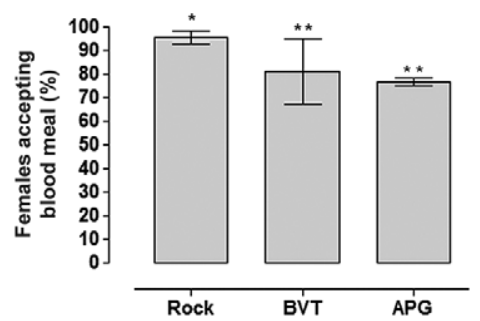

B

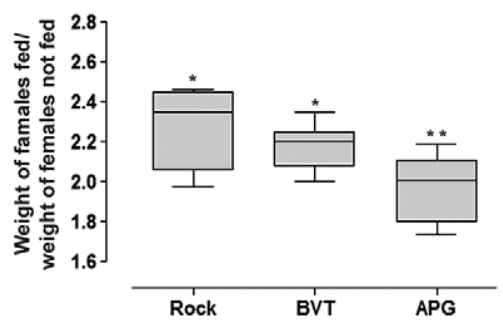

Fig 3: rate of blood meal acceptance (A) and amount of ingested blood (B) by Rockefeller (Rock), Aparecida de Goiânia (APG) and Boa Vista (BVT) Aedes aegypti females. Asterisks above columns mean significant differences $(\mathrm{p}<0.05)$ 
resistance to OPs. Adult starvation tolerance was also evaluated in an Ae. aegypti strain selected with Bacillus thuringiensis var. israelensis toxins. In this case, no differences were apparent between the selected group and the control group (Paris et al. 2011).

Under our experimental conditions, fewer field population females accepted the blood meal when compared to the Rock strain. Additionally, females from Aparecida de Goiânia, the population with a higher temephos resistance level, ingested $15 \%$ less blood than Rock females and, consequently, laid $21 \%$ fewer eggs. This is a consistent result because the amount of blood ingested by culicids is directly related to the number of eggs deposited (Clements 1992). A similar situation was observed by Martins et al. (2012) working with females after pyrethroid selection. To our knowledge, there are no previous reports for culicids dealing specifically with insecticide resistance influence over the acceptance of blood meal.

Kumar et al. (2009) studied the effect of deltamethrin selection on the fitness of an Ae. aegypti field population, with both larvae and adults. The number and viability of eggs laid per female were evaluated in the course of three gonotrophic cycles. A similar investigation was conducted by Kumar and Pillai (2011) with $C x$. quinquefasciatus. In both cases, no significant differences were noted in the number of blood-fed females between control and insecticide-selected groups. However, in all cases, when compared to controls, a higher number of pyrethroid selected females accepted the blood meal upon the first offer. Nevertheless, pyrethroid-selected females from both species laid fewer eggs than control females. Although the amount of ingested blood was not evaluated, the number of eggs laid is an indirect measurement of this parameter (Clements 1992) and it is likely that pyrethroid-selected females consumed lower amounts of blood. In all cases, the viability of eggs laid by deltamethrin-selected females was also lower than that of susceptible females. Accordingly, deltamethrin-resistant Cx. pipiens pallens females laid fewer eggs than susceptible females, though in this case, viability differences were not noted (Li et al. 2002). Likewise, we did not find differences in egg viability for APG or BVT populations when compared to the Rock strain.

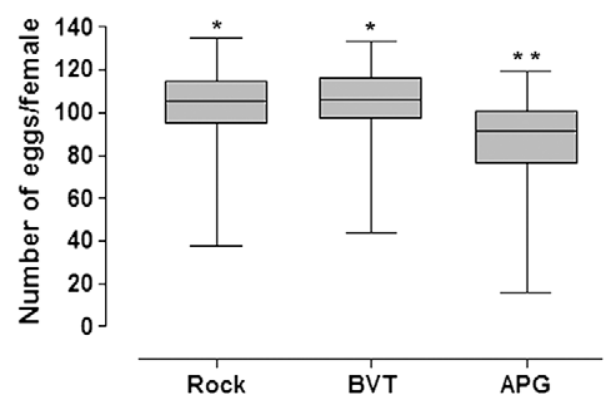

Fig. 4: median number of eggs laid by Rockefeller (Rock), Aparecida de Goiânia (APG) and Boa Vista (BVT) Aedes aegypti females. Asterisks mean significant differences $(*: \mathrm{p}<0.05 ; * *: \mathrm{p}<0.01)$.
One of the most affected parameters was the mating efficiency of each population. After three days, almost all Rock males (98\%) successfully inseminated their three available females. In contrast, the performance of males from field populations was much lower; only 54\% of BVT and 7\% of APG males inseminated their three females. Mating efficiency was inversely proportional to the temephos resistance ratio. Using a different approach, Berticat et al. (2002) demonstrated that OP resistance significantly affects the insemination rate of $C x$. pipiens, resistant males being less competitive. Therefore, the majority of females were inseminated by susceptible males.

Although OPs and pyrethroids have been adopted in Brazil for the control of Ae. aegypti for more than four decades, there are few studies regarding the effects of these insecticides on vectorial capacity, particularly in field populations. Different aspects of insect biology, such as their development, reproduction or longevity, can be affected by insecticide resistance; the extent of these effects can depend not only upon the type and amount of insecticide used and the frequency of its application, but also on the resistance mechanism involved. Field populations can be exposed to different insecticides as a result of domestic or agricultural applications. Insect field populations can also be subjected to eventual non-integrated insecticide use when different disease vectors coexist in a given locality. Hence, perturbation of a given vector biology parameter can result from different selected mechanisms operating simultaneously. Therefore, the fitness cost related to resistance will ultimately depend upon the previous insecticide exposure of each vector population.

Although it is certain that insecticide resistance can alter vector biology, evaluation of individual parameters by different authors can reveal conflicting results. These differences may be attributed to peculiarities of the various vector species with respect to the effects of distinct insecticides or insecticide classes, differences in the genetic background of field populations leading to activation of a variety of potential resistance mechanisms or even to variations in the methodologies adopted in the evaluation of these effects. Moreover, it is important to take into account that insect fitness aspects are generally evaluated under optimal laboratory conditions. Therefore, it is expected that the extent of the effects analysed is underes-

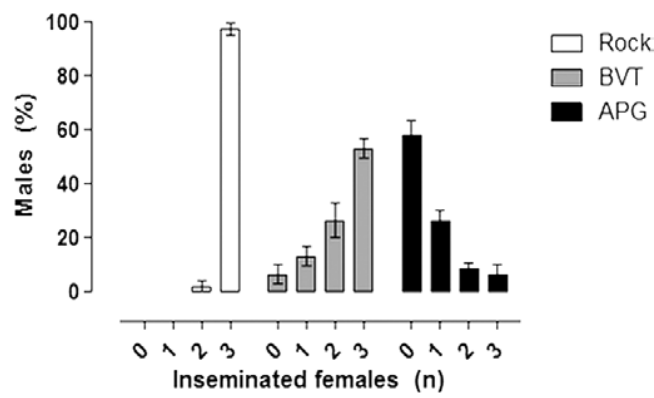

Fig. 5: mating effectiveness of Rockefeller (Rock), Aparecida de Goiânia (APG) and Boa Vista (BVT) Aedes aegypti specimens under confined conditions. In all cases, tubes containing one virgin male and three virgin females were kept for three days. Spermathecae of all females were then examined for the presence of sperm. 
timated in the laboratory, where insects are not exposed to more stringent conditions, such as abrupt temperature changes, limited nutrition and predation. In contrast, in the field, insecticide resistance-fitness trade-offs can lead to more severe consequences (Kishony \& Leibler 2003, Hardstone et al. 2009). Additionally, different alleles related to resistance can affect fitness positively or negatively, depending upon various interactions and the environment to which insects are subjected (Berticat et al. 2008).

It must be noted that both populations evaluated in this study were also resistant to the pyrethroid deltamethrin and that $k d r$ mutations in the target site of this insecticide, i.e., the voltage-gated sodium channel, were detected. Previous reports suggest that these mutations spread rapidly among insect field populations (Garcia et al. 2009, Martins et al. 2009). In Ae aegypti, this is likely related to the small impact of $k d r$ on fitness, as determined through evaluations conducted in an insecticidefree environment under optimal laboratory conditions (LPBO Souza, unpublished obervations). According to our results, resistance to the OP temephos is directly related to the decreased viability and reproductive performance of the field populations compared to the Rock control strain. However, since the strains tested originated from the field, it is likely that other mechanisms involved in resistance to insecticides and $k d r$ mutations may be contributing to the effects observed on fitness.

Because the application of chemical insecticides still plays an important role in the control of several insect vectors, a detailed analysis of the effect of resistance on the biology of these species can directly contribute to the development of novel control strategies as well as to the management of resistance in natural vector populations.

\section{ACKNOWLEDGEMENTS}

To Gilberto Couto Alcântara, for technical assistance, to Heloisa Maria Nogueira Diniz (Serviço de Produção e Tratamento de Imagem/IOC), for help with the figures, to Dr José Bento Pereira Lima, for critical evaluation of the bioassays, and to Mitchell Raymond Lishon (UCLA), for English revision.

\section{REFERENCES}

Agnew P, Berticat C, Bedhomme S, Sidobre C, Michalakis Y 2004. Parasitism increases and decreases the costs of insecticide resistance in mosquitoes. Evolution 58: 579-586.

Belinato TA, Martins AJ, Lima JBP, Lima-Camara TN, Peixoto AA, Valle D 2009. Effect of the chitin synthesis inhibitor triflumuron on the development, viability and reproduction of Aedes aegypti. Mem Inst Oswaldo Cruz 104: 43-47.

Berticat C, Bonnet J, Duchon S, Agnew P, Weill M, Corbel V 2008. Costs and benefits of multiple resistance to insecticides for Culex quinquefasciatus mosquitoes. BMC Evol Biol 8: 104.

Berticat C, Boquien G, Raymond M, Chevillon C 2002. Insecticide resistance genes induce a mating competition cost in Culex pipiens mosquitoes. Genet Res 79: 41-47.

Berticat C, Duron O, Heyse D, Raymond M 2004. Insecticide resistance genes confer a predation cost on mosquitoes, Culex pipiens. Genet Res 83: 189-196.

Braga IA, Lima JBP, Soares SS, Valle D 2004. Aedes aegypti resistance to temephos during 2001 in several municipalities in the states of Rio de Janeiro, Sergipe and Alagoas, Brazil. Mem Inst Oswaldo Cruz 99: 199-203.
Braga IA, Valle D 2007a. Aedes aegypti: histórico do controle no Brasil. Epidemiol Serv Saude 16: 113-118.

Braga IA, Valle D 2007b. Aedes aegypti: vigilância, monitoramento da resistência e alternativas de controle no Brasil. Epidemiol Serv Saude 16: 295-302.

Chavasse DC, Yap HH 1997. Chemical methods for the control of vectors and pests of public health importance. WHO/CTD/ WHOPES/97.2. Available from: whqlibdoc.who.int/hq/1997/ WHO_CTD_WHOPES_97.2.pdf.

Clements AN 1992. The biology of mosquitoes. Development, nutrition and reproduction, Vol. 1, Chapman \& Hall, London, 509 pp.

da-Cunha MP, Lima JBP, Brogdon WG, Moya GE, Valle D 2005. Monitoring of resistance to the pyrethroid cypermethrin in Brazilian Aedes aegypti (Diptera: Culicidae) populations collected between 2001 and 2003. Mem Inst Oswaldo Cruz 100: 441-444.

Davidson G, Zahar AR 1973. The practical implications of resistance of malaria vectors to insecticides. Bull WHO 49: 475-483.

García PG, Flores AE, Fernández-Salas I, Saavedra-Rodríguez K, Reyes-Solis G, Lozano-Fuentes S, Bond JG, Casas-Martínez M, Ramsey JM, García-Rejón J, Domínguez-Galera M, Ranson H, Hemingway J, Eisen L, William C, Black WC 2009. Recent rapid rise of a permethrin knock down resistance allele in Aedes aegypti in Mexico. PLoS Negl Trop Dis 3: e53.

Gubler DJ 2002. Epidemic dengue/dengue hemorrhagic fever as a public health, social and economic problem in the 21 st century. Tr Microbiol 10: 100-103.

Hardstone MC, Huang X, Harrington LC, Scott JG 2010. Differences in development, glycogen and lipid content associated with cytochrome $\mathrm{P} 450$-mediated permethrin resistance in Culex pipiens quinquefasciatus (Diptera: Culicidae). J Med Entomol 47: 188-198.

Hardstone MC, Lazzaro BP, Scott JG 2009. The effect of three environmental conditions on the fitness of cytochrome P450 monooxygenase-mediated permethrin resistance in Culex pipiens quinquefasciatus. BMC Evol Biol 9: 42.

Hawk CT, Leary SL 1995. Formulary for laboratory animals, Iowa State University Press, Iowa, $101 \mathrm{pp}$.

Hemingway J, Ranson H 2000. Insecticide resistance in insect vectors of human disease. Ann Rev Entomol 45: 371-391.

Jansen CC, Beebe NW 2010. The dengue vector Aedes aegypti: what comes next. Microbes Infect 12: 272-279.

Kishony R, Leibler S 2003. Environmental stresses can alleviate the average deleterious effect of mutations. J Biol 2: 14.

Kumar S, Pillai MKK 2011. Correlation between the reproductive potential and the pyrethroid resistance in an Indian strain of filarial vector, Culex quinquefasciatus Say (Diptera: Culicidae). Bull Entomol Res 101: 25-31.

Kumar S, Thomas A, Samuel T, Sahgal A, Verma A, Pillai MKK 2009. Diminished reproductive fitness associated with the deltamethrin resistance in an Indian strain of dengue vector mosquito, Aedes aegypti L. Trop Biomed 26: 155-164.

Kuno G 2010. Early history of laboratory breeding of Aedes aegypti (Diptera:Culicidae) focusing on the origins and use of selected strains. J Med Entomol 47: 957-971.

Li X, Ma L, Sun L, Zhu C 2002. Biotic characteristics in the deltamethrin-susceptible and resistant strains of Culex pipiens pallens (Diptera: Culicidae) in China. Appl Entomol Zool 37: 305-308.

Lima JBP, da-Cunha MP, Silva-Jr RC, Galardo AKR, Soares SS, Braga IA, Ramos RP, Valle D 2003. Aedes aegypti resistance to organophosphates in several localities in Rio de Janeiro and Espírito Santo, Brazil. Am J Trop Med Hyg 68: 329-333. 
Mackenzie JS, Gubler DJ, Petersen LR 2004. Emerging flaviviruses: the spread and resurgence of Japanese encephalitis, West Nile and dengue viruses. Nat Med 10 (Suppl. 12): S98-S109.

Macoris M, Andrighetti MT, Takaku L, Glasser CM, Garbeloto VC, Cirino VC 1999. Changes in susceptibility of Aedes aegypti to organophosphates in municipalities in the state of São Paulo, Brazil. Rev Saude Publica 33: 521-522.

Martins AJ, Lima JBP, Peixoto AA, Valle D 2009. Frequency of Val1016Ile mutation in the voltage-gated sodium channel gene of Aedes aegypti Brazilian populations. Trop Med Int Health 14: 1351-1355.

Martins AJ, Ribeiro CDM, Bellinato DF, Peixoto AA, Valle D, Lima JBP 2012. Effect of insecticide resistance on development longevity and reproduction of field or laboratory selected Aedes aegypti populations. PloS ONE 7: e31889.

Martins AJ, Valle D 2012. The pyrethroid knockdown resistance. In M Larramendy, S Soloneski (eds.), Book 2, Insecticides, Intech, La Plata, p. 17-38.

Montella IR, Martins-Jr AJ, Viana-Medeiros PF, Lima JBP, Braga IA, Valle D 2007. Insecticide resistance mechanisms of Brazilian $\mathrm{Ae}$ des aegypti populations from 2001 to 2004. Am J Trop Med Hyg 77: 467-477.

Paris M, David J, Despres L 2011. Fitness costs of resistance to Bti toxins in the dengue vector Aedes aegypti. Ecotoxicology 20: 1184-1194.

Ponlawat A, Harrington LC 2005. Blood feeding patterns of Aedes aegypti and Aedes albopictus in Thailand. J Med Entomol 42: 844-849.
Ranson H, N'Guessan R, Lines J, Moiroux N, Nkuni Z, Corbel V 2004. Pyrethroid resistance in African anopheline mosquitoes: what are the implications for malaria control? Trends Parasitol 27: 91-98.

Raymond M 1985. Presentation d'une programme d'analyse logprobit pour microordinateur cahiers Orstrom. Ser Ent Med Parasitol 22: 117-121.

Rivero A, Vézilier J, Weill M, Read AF, Gandon S 2010. Insecticide control of vector-borne diseases: when is insecticide resistance a problem? PLoS Pathog 6: e1001000.

Roush RT, McKenzie JA 1987. Ecological genetics of insecticide and acaricide resistance. Annu Rev Entomol 32: 361-380.

Siriyasatien P 2010. Identification of blood meal of field caught Aedes aegypti (L.) by multiplex PCR. Southeast Asian J Trop Med Public Health 41: 43-47.

Valencia MDP, Miller LH, Mazur P 1996. Permeability of intact and dechorionated eggs of the Anopheles mosquito to water vapour and liquid water: a comparison with Drosophila. Cryobiol 33: 142-148.

WHO 1981. Instructions for determining the susceptibility or resistance of mosquito larvae to insecticides. WHO/VBC/81.807.1981.Available from: whqlibdoc.who.int/temp/WHO_VBC_81.807.pdf.

WHO 1998. Test procedures for insecticide resistance monitoring in malaria vectors, bio-efficacy and persistence of insecticides on treated surfaces. WHO/CDS/CPC/MAL/98.12. Available from: who.int/malaria/publications/atoz/who_cds_cpc_mal_98_12/en/ index.html. 